\title{
Editorial: Contributions of Behavior and Physiology to Conservation Biology
}

\author{
Caitlin R. Gabor ${ }^{1 *}$ and Susan C. Walls ${ }^{2}$ \\ ${ }_{1}^{1}$ Texas State University, San Marcos, TX, United States, ${ }^{2}$ United States Geological Survey, Wetland and Aquatic Research \\ Center, Gainesville, FL, United States
}

Keywords: behavior, physiology, conservation management, environmental stressors, urbanization

\section{Editorial on the Research Topic}

\section{Contributions of Behavior and Physiology to Conservation Biology}

Conservation biology is a rapidly evolving discipline, with its synthetic, multidisciplinary framework expanding extensively in recent years. Seemingly disparate disciplines, such as behavior and physiology, are being integrated into this discipline's growing portfolio, resulting in diverse tools that can help develop conservation solutions. Behavior and physiology have traditionally been considered separate fields, yet their integration can provide a more comprehensive approach to developing solutions to conservation and management problems. However, demonstrations are needed of how behavior and physiology-either separately or combined-have contributed to conservation success. Examining species' vulnerabilities to extinction through the lenses of behavior and physiology can provide insight into the mechanisms that drive population declines and extirpations. Our goal is to increase awareness of the benefit of combining behavioral and physiological tools to improve conservation management decisions. Such studies can also help strengthen the basis for evidence-based conservation which, in some cases, has been previously lacking. The diverse studies in our Research Topic illustrate key examples of ways that behavior and physiology can be incorporated into conservation biology. Three main themes emerged from the invited papers with respect to their relevance to conservation: (1) Stress physiology, (2) indicators of health and disease dynamics, (3) and movement ecology. But these themes were also intertwined, thus showing the importance of integrating multiple fields of research to successfully address questions about conservation biology.

Two mini reviews discuss the importance of examining how stress physiology may affect individual fitness and capacity to cope with change which, ultimately, affects the resiliency of populations. Walls and Gabor, in their mini review, promoted combining studies of behavior and physiology to aid in developing conservation strategies for amphibians, which could provide conservation managers with workable solutions to global environmental change. Walls and Gabor also pointed out that studies of behavior are useful to understand how native amphibian species respond to invasive predators. This is supported by Roznik et al. who combined physiology and behavior for a more holistic understanding of the impacts of competition between native and invasive frogs. These authors found that invasive Cuban tree frogs outperformed two native species in jump length and speed, offsetting the costs of dehydration. This work also ties in well to a review 
by Joly, who indicated the importance of exploring movement ecology (on multiple spatial scales) of invasive species to provide insights for invasive species management. Walls and Gabor also emphasized that more studies on phenological shifts of species in response to recent climate change are needed, as explored by Bókony et al. with birds. These authors found that bird species varied their migratory behaviors in response to contemporary climate change which, in turn, influenced subsequent trends in population size.

Walls and Gabor further promoted the value of examining multiple measures of physiological health to provide a more holistic assessment of an organism's response to environmental change and overall population resilience. Amdekar et al. did just that in their study of lizards. These authors explored multiple markers of health and found no differences in body condition, number of ectoparasites and a measure of immune response between urban and rural populations. However, Amdekar et al. found that urban lizards, compared to rural ones, had lower heterophil to lymphocyte ratios, along with lower testosterone levels-two key measures of physiological health. This research highlights the importance of using multiple physiological measures to explore the health of populations in rapidly changing environments. Last, Walls and Gabor emphasized the contribution that behavioral studies can make to mitigating disease outbreaks. Hettyey et al. provided an example of importance of such studies: these authors suggested that, by providing localized heat sources in situ, behavioral temperature preferences, for the heat source, could minimize transmission of the fungal pathogen Batrachochytrium dendrobatidis.

In the other mini-review, Narayan et al. focused on multiple non-invasive methods for measuring biomarkers of stress in amphibians, a research gap pointed out by Walls and Gabor. The ability to measure stress non-invasively provides a mechanism for detecting problems in populations before endangerment occurs as it allows one to explore the capacity of a population to cope with a changing environment. The use of such methods allows for studies of imperiled amphibians where destructive measures of physiology are not viable and will aid in understanding the link between population declines and physiological responses to altered environments. Gabor et al. provided an example of the usefulness of non-invasive water-borne hormones to understand mechanisms associated with amphibian population declines. They examined the behavioral and physiological changes associated with exposure to Roundup ${ }^{\circledR}$, a common pollutant (glyphosate) in aquatic environments that are near agricultural and urban areas. These authors found that exposure to Roundup ${ }^{\circledR}$ may prevent adaptive antipredator responses in larval amphibians but does not affect endogenous corticosterone levels.

Many of the studies in our Research Topic found that examining aspects of movement behavior can help promote conservation outcomes as anthropogenic factors dramatically alter natural ecosystems. For example, McEntire and Maerz, using agent-based models, found that plant climbing behavior in salamanders may help mitigate a species' sensitivity to climate. Bredeweg et al. directly tested movement behavior of metamorphic frogs associated with pond drying and dry habitat conditions. These authors found that larger individuals (from permanent ponds) were more likely to move, and to move further, than smaller individuals, indicating that smaller individuals may not be able to overcome drought conditions by dispersing. As with Bókony et al., there is some indication that organisms are showing adaptive changes in behavior and Bredeweg et al. showed that these behaviors may minimize population declines.

Rapidly changing environments are potentially exposing organisms to unique challenges by generating stressors that affect physiology, behavior, and locomotor efficiency. Understanding how organisms respond to stressors can provide insight into whether populations are able to potentially adapt to these environmental changes. Kern and Langerhans found that fish from urban streams showed higher locomotor efficiency when swimming compared to fish from rural populations, where water velocities are lower during rain events. These authors also found that differences in swimming efficiency in urban populations were the result of genetic evolution, not phenotypic plasticity, and that urban populations were less plastic in their locomotor efficiency than rural populations. These results, together with those of Amdekar et al. and Roznik et al., provide insights into the consequences of urbanization, yet information is still lacking on whether such responses protect these species from population decline or extirpation.

In a more applied setting, Settle et al. explored reproductive behavior of the endangered Ozark hellbender in captivity. These authors found a rapid behavioral change associated with oviposition. This was followed by additional reproductive events in other females that were presumably stimulated by an increase in pheromones/reproductive hormones in the water. Further studies on the physiology of Ozark hellbenders may inform the use of hormones to trigger mating, thus enhancing captive breeding success.

Addressing the biodiversity crisis is inherently complicated because of the multitude of environmental drivers of population declines. Integrating tools and techniques from various disciplines is a more holistic approach in conservation planning and targets this complexity by revealing the mechanisms by which various stressors may be contributing to declines. The articles in this Research Topic provide examples of how studies of behavior and physiology can be integrated into conservation decision-making and strategic planning in a variety of taxa. By highlighting such work, our hope is that others in the conservation community will be motivated to adopt similar approaches, thus allowing a greater understanding of the mechanisms behind population declines and factors that may aid or hinder the future survival of some species.

\section{AUTHOR CONTRIBUTIONS}

CG and SW contributed equally to the proposal and editorial work of this Research Topic. Both CG and SW equally contributed to the writing of the Editorial. 


\section{ACKNOWLEDGMENTS}

We thank all guest editors and reviewers that evaluated the 12 contributions of this Research Topic. Any use of trade, firm, or product names is for descriptive purposes only and does not imply endorsement by the U.S. Government. This work is ARMI contribution number 726 .

Conflict of Interest: The authors declare that the research was conducted in the absence of any commercial or financial relationships that could be construed as a potential conflict of interest.
Copyright (c) 2020 Gabor and Walls. This is an open-access article distributed under the terms of the Creative Commons Attribution License (CC BY). The use, distribution or reproduction in other forums is permitted, provided the original author(s) and the copyright owner(s) are credited and that the original publication in this journal is cited, in accordance with accepted academic practice. No use, distribution or reproduction is permitted which does not comply with these terms. 